\title{
The relationship between nasal polyps, bronchial asthma, allergic rhinitis, atopic dermatitis, and non-allergic rhinitis
}

\author{
Oksana Wojas ${ }^{1}$, Magdalena Arcimowicz ${ }^{2}$, Konrad Furmańczyk ${ }^{1,3}$, Adam Sybilski ${ }^{4}$, Filip Raciborski ${ }^{1}$, \\ Aneta Tomaszewska ${ }^{1}$, Artur Walkiewicz ${ }^{1}$, Piotr Samel-Kowalik ${ }^{1}$, Bolesław Samoliński ${ }^{1}$, Edyta Krzych-Fatta ${ }^{1}$
}

${ }^{1}$ Department of Prevention of Environmental Hazards and Allergology, Faculty of Health Sciences, Medical University of Warsaw, Warsaw, Poland

${ }^{2}$ Department of Otorhinolaryngology Head and Neck Surgery, Medical University of Warsaw, Warsaw, Poland

${ }^{3}$ Applied Mathematics, Faculty of Applied Informatics and Mathematics, Warsaw University of Life Sciences (SGGW), Warsaw, Poland ${ }^{4}$ Second Department of Paediatrics, Centre of Postgraduate Medical Education, Warsaw, Poland

Adv Dermatol Allergol 2021; XXXVIII (4): 650-656

DOI: https://doi.org/10.5114/ada.2020.94400

\begin{abstract}
Introduction: Nasal polyps are frequently associated with bronchial asthma and rhinitis. The chronic nature of the symptoms, the high post-treatment recurrence rates, as well as various comorbidities, constitute key factors that significantly affect the quality of life of patients diagnosed with this condition.

Aim: The purpose of the study was to estimate the prevalence of nasal polyps in the examined population and to assess the possible associative occurrence of nasal polyps (NP) with bronchial asthma (BA), allergic (AR) and nonallergic rhinitis (NAR), and atopic dermatitis (AD).

Material and methods: The ECRHS II and ISAAC questionnaires of the study group of 18,458 individuals, including 4,473 6-7-year-olds (24.2\%), 4,675 13-14-year-olds (25.4\%), and 9,310 20-44-year-olds (50.4\%) were performed. Results: The prevalence of nasal polyps in the examined group was $1.1 \%$, reported by a total of 204 individuals. Nasal polyps were reported more frequently among urban residents (191 (1.1\%)) than rural residents (13 (0.6\%)). Our study demonstrated a correlation between the presence of nasal polyps and asthma, as well as allergic and non-allergic rhinitis The greatest risk factor for NP in the evaluated subpopulation with multiple allergic conditions was the co-existence of non-allergic rhinitis and atopic dermatitis ( $\mathrm{OR}=6.09 ; 95 \% \mathrm{Cl}: 3.4-10.93)$.

Conclusions: Nasal polyps are relatively rare in the evaluated Polish population. Nonetheless, we believe their cooccurrence with non-allergic rhinitis, allergic rhinitis, bronchial asthma, and atopic dermatitis to be of significant importance, as it illustrates the phenomenon of multimorbidity of inflammatory conditions affecting the upper and lower respiratory tract.
\end{abstract}

Key words: nasal polyps, multimorbidity, epidemiology, prevalence, comorbidity.

\section{Introduction}

Nasal polyps (NP) are a chronic inflammatory condition of the upper respiratory tract that occur exclusively among humans; they have a considerable negative impact on the quality of life of affected individuals and exhibit a strong recurrent tendency regardless of treatment. The European Position Paper on Rhinosinusitis and Nasal Polyps (EPOS 2012) classifies NP as a subtype of chronic rhinosinusitis and therefore recommends the terminology of 'chronic rhinosinusitis with nasal polyps' (CRSwNP) [1]. The general prevalence of NP is $1-4 \%$ in adults and approximately $0.5 \%$ in children $[1,2]$. Based on epidemiological studies, the prevalence of CRSwNP was estimated at $2.1 \%$ in France, $4.4 \%$ in Finland, and approx- imately $4.2 \%$ in the USA [3-5]. According to EPOS-based surveys conducted in China, the prevalence of CRSwNP was estimated at $2.2-8 \%$ [6].

Due to a number of comorbidities associated with $N P$, it is yet to be conclusively determined whether NP are solely a localised pathology or local manifestations of a multifactorial, systemic disease. Bronchial asthma and rhinitis are commonly cited as conditions that frequently coexist with CRSWNP [1, 7]. In particular, a large retrospective study conducted by Tan et al., evaluating over 400,000 primary care patients, showed that those diagnosed with CRSwNP had a significantly higher premorbid prevalence of acute rhinosinusitis, allergic rhinitis, chronic rhinitis, and asthma [8]. The issue of multimorbidity, or

Address for correspondence: Magdalena Arcimowicz MD, PhD, Department of Otorhinolaryngology Head and Neck Surgery, Medical University of Warsaw, 1a Banacha St, 02-097 Warsaw, Poland, phone: +48 2259925 21, fax: +48 225992523 , e-mail: marcimowicz.wum@gmail.com Received: 15.03 .2020 , accepted: 25.03 .2020$. 
multiple morbidities, has been extensively discussed in contemporary allergy- and rhinology-oriented literature. Bousquet et al. described the phenomenon of multimorbidity in the case of allergic conditions including allergic rhinitis, bronchial asthma, and atopic dermatitis, emphasising that these conditions are characterised by similar underlying disorders, both non-immunological and immunological (mainly polysensitisation, i.e. sensitisation to multiple allergens) [9]. A study by Raciborski et al. supported the association of multimorbidity with polysensitisation, further demonstrating that bronchial asthma is a greater risk factor for developing multimorbidity than allergic rhinitis and atopic dermatitis [10].

The underlying mechanisms that contribute to the chronic sinonasal inflammation observed in CRSWNP have not yet been fully explained, which is one of the reasons for the limited variety of therapeutic options available to patients with CRSwNP. Topical glucocorticoids administered intranasally remain the treatment of choice and are recommended as first-line therapy, as well as a means to alleviate inflammation and recurrences, while in certain cases a short-term, adjunctive oral corticosteroid treatment may be implemented. Patients with severe rhinosinusitis and those who do not respond to conservative therapeutic options should be considered for sinus surgery. Furthermore, a number of ongoing studies are evaluating the use of biological drugs (omalizumab, mepolizumab, and dupilumab) in the treatment of NP [1, 9-11].

\section{Aim}

The objective of our study was to estimate the prevalence of nasal polyps in the study population of ECAP (Epidemiology of Allergic Diseases in Poland) and to assess the eventual relationships between the occurrence of NP and bronchial asthma (BA), allergic rhinitis (AR), non-allergic rhinitis (NAR), and atopic dermatitis (AD).

\section{Material and methods}

The ECAP study was a continuation of the European Community Respiratory Health Survey II (ECRHS II) [12], while its design was also based on the research goals and methodology of the International Study of Asthma and Allergies in Childhood (ISAAC) [13]. The ECAP study was part of research project No. 6 PO5 2005 C/06572 "Implementation of the system for the prevention and early detection of allergies in Poland", commissioned by the Polish Minister of Health. The ECAP study was to be conducted in a population of adults aged 20-44 years (conforming to the ECRHS protocol), as well as two child populations aged 6-7 and 13-14 years (conforming to the ISAAC protocol).

Our survey study encompassed a total of 20,545 respondents. Out of the 20,454 completed questionnaires, 18,458 passed the final quality assessment, including
9922 (53.75\%) female respondents and 8536 (46.25\%) male respondents. The remaining questionnaires exhibited significant irregularities during quality assessment and were subsequently excluded from further analyses. The age subpopulations of younger children aged 67 years, older children aged 13-14 years, and adults aged 20-44 years, constituted respectively $24.2 \%, 25.4 \%$, and $50.4 \%$ of the population evaluated during the main part of the study.

In order to meet the research goals, the areas to be examined were selected via purposive sampling, allowing all the essential factors of the study to be taken into account. Analysis of the research subject led to the establishment of three additional city selection criteria: geographic location, based on the pre-identified regional variability of pollination periods, resulting in differences of air-borne allergen concentration; population of cities; and severity of air pollution and climate variation. The following cities were selected based on the aforementioned factors: Warsaw, Lublin, Bialystok, Gdansk, Poznan, Wroclaw, Katowice, and Krakow, with the addition of one rural area consisting of Zamosc and Krasnystaw counties, with the towns of Zamosc and Krasnystaw excluded from the analysis. The examined areas were selected via purposive (non-probability) sampling, whereas respondents from those regions were selected via random sampling. The random selection method was developed to ensure a representative study sample for the given population, and the database of the Polish National Identification Number (PESEL) was used employed, containing information on given and family names, address, age, and sex of Polish citizens, as its sampling frame.

In order to determine the corresponding declared diagnoses, the following questionnaire items were selected: item v176: Do you suffer from any nasal allergies, including hay fever? (AR); item v176: Have you ever suffered from asthma? (BA); item v191: Have you ever had eczema (atopic dermatitis) or any other type of skin allergy? (AD); and item v199: Do you have nasal polyps? (NP). In accordance with the research objectives of the study, we established the selection criteria based on affirmative responses to questions addressing NP, AR, NAR, BA, and $A D$, also factoring in the area of residence and tobacco consumption. Our analyses reflected study-group stratification by age, sex, economic status, and the level of education.

\section{Statistical analysis}

The $\chi^{2}$ statistic and the odds ratio (OR) with a $95 \%$ confidence interval were calculated to determine the strength of relationship and correlation between the evaluated variables. The results were considered statistically significant at $p<0.05$. The study had been approved by both the Institutional Review Board at the Medical University of Warsaw (decision No. KB/206/2005) and the General Inspector for the Protection of Personal Data. 


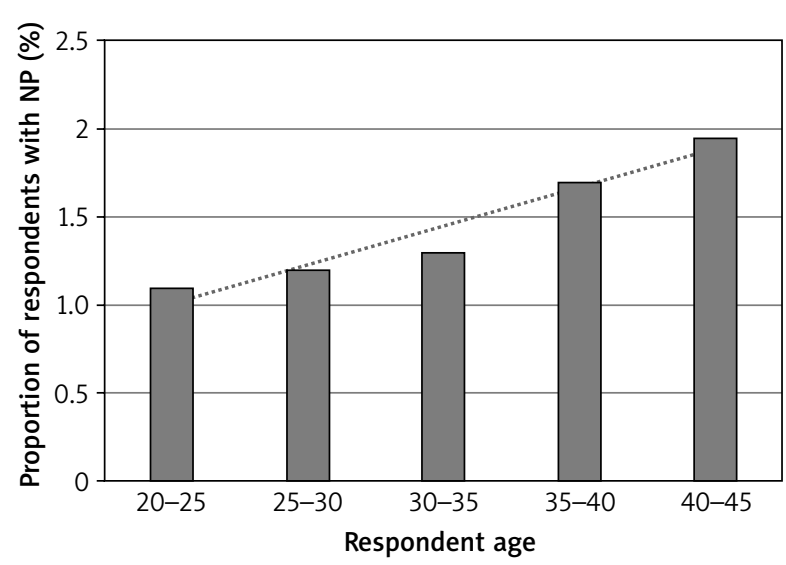

Figure 1. Prevalence of nasal polyps with respect to the age of respondents

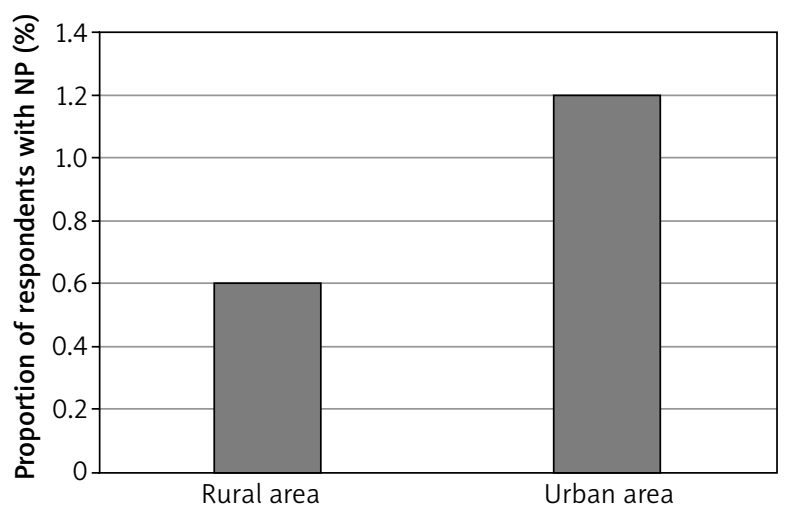

Figure 2. The regional variability of NP prevalence in rural and urban areas (Zamosc and Krasnystaw counties vs. the city of Lublin)

\section{Results}

The prevalence of nasal polyps in the analysed Polish population was assessed based on the adopted criteria of age, sex, and area of residence, accompanied by an estimation of concomitant risk of non-allergic rhinitis, allergic rhinitis, bronchial asthma, and atopic dermatitis. The analysis of selected risk factors revealed no significant impact of traffic congestion (exhaust fumes exposure), residence size, or economic status on NP prevalence.

The frequency of nasal polyp occurrence was correlated with at least 1 year of tobacco consumption, while no statistically significant correlation was observed between exposure to tobacco smoke (i.e. passive smoking) and the occurrence of nasal polyps $(\mathrm{OR}=1.5 ; 95 \% \mathrm{Cl}$ : $1.081-2.102 ; p=0.015)$.

A total of 204 (1.1\%) out of the 18,458 respondents reported nasal polyps, with the following age stratification (Figure 1):

- 29 of the $44736-7$-year-olds, including 17 females (0.8\%) and 12 males (0.05\%);
- 31 of the 4675 13-14-year-olds, including 10 females (0.4\%) and 21 males (0.9\%);

- 144 of the 9310 20-44-year-olds, including 77 females (1.4\%) and 67 males (1.7\%).

Our data exhibited no significant variability in NP prevalence between these age groups. Notably, a linear increase of prevalence with age was observed, with the number of respondents who reported NP increasing with a mean of $0.05 \%$ per year of age $\left(R^{2}=0.92\right)$.

There was a significant discrepancy between rural and urban residents, the declared NP prevalence among rural respondents being $13(0.6 \%)$ of the 2034 , compared to $191(1.1 \%)$ of the 16424 urban respondents ( $p=0.043)$ (Figure 2). The proportions of NP prevalence in the evaluated areas ranged from $0.6 \%$ in the rural, south-east county of Zamosc to $1.4 \%$ in the cities of Gdansk and Warsaw, located in northern and central Poland.

The prevalence of NP was higher for all respondents with non-allergic rhinitis, regardless of age. This relationship was particularly pronounced and statistically significant among 13-14-year-olds and 20-44-year-olds. We observed a 2.5 -fold increase $(p<0.001)$ of concomitant NP risk in those with AR, and a nearly 6 -fold higher risk in those with NAR ( $p<0.001)$, compared to respondents without AR. Moreover, the risk of concomitant NP was 3.5 times higher in respondents with BA when compared to those without BA $(p<0.001)$. All age groups displayed a tendency for concomitant NP with symptomatic $A D$, a correlation statistically significant solely among adults (Figure 3).

The subgroups with the highest rates of co-existing allergic and non-allergic conditions, with the exclusion of nasal polyps, were identified as NAR + BA and NAR + AD, estimated at nearly 3.4\%. Conversely, the groups displaying the lowest rates were, respectively: $B A+A D$ at $0.4 \%$, $\mathrm{NAR}+\mathrm{BA}+\mathrm{AD}$ at $0.3 \%$, and $\mathrm{AR}+\mathrm{BA}+\mathrm{AD}$ at $0.3 \%$ of all respondents. The proportion of concomitant NP in the groups with allergic and non-allergic conditions ranged from $3 \%$ to $6 \%$, with the highest prevalence of NP in the $\mathrm{NAR}+\mathrm{BA}+\mathrm{AD}$ group (estimated at 5.5\%) and in the AR $+B A+A D$ group (4\%) (Table 1 A). The greatest risk factor for NP in the evaluated subpopulation with multiple allergic conditions was identified as the co-existence of NAR and AD (OR =6.09; 95\% Cl: 3.4-10.93). Another combination of allergic conditions significantly increasing the risk of NP was $A R+B A+A D(O R=5.51 ; 95 \% \mathrm{Cl}$ : 1.71-17.8) (Table 1 B).

\section{Discussion}

The ECAP study focused on the overall epidemiology of allergic conditions in Poland, examining the associated comorbidities and underlying factors that may affect the development thereof. The ECAP study encompassed the subject of nasal polyps; however, the self-reported prevalence of the condition was limited to the question Do you 
Table 1. Co-existence of allergic and non-allergic conditions

A. Co-existence of nasal polyps in subgroups with multiple allergic and non-allergic conditions

\begin{tabular}{lcccccc}
\hline \multicolumn{7}{c}{ Proportions of co-existing allergic and non-allergic conditions (excluding cases of NP) } \\
\hline $\begin{array}{l}\text { NAR and BA } \\
(n=604)\end{array}$ & $\begin{array}{c}\text { NAR and AD } \\
(n=402)\end{array}$ & $\begin{array}{c}\mathrm{BA} \text { and AD } \\
(n=65)\end{array}$ & $\begin{array}{c}\mathrm{BA} \text { and } \mathrm{AR} \\
(n=482)\end{array}$ & $\begin{array}{c}\mathrm{AD} \text { and AR } \\
(n=285)\end{array}$ & $\begin{array}{c}\text { NAR and BA } \\
\text { and AD }(n=52)\end{array}$ & $\begin{array}{c}\text { AR and BA } \\
\text { and AD }(n=2)\end{array}$ \\
\hline $3.39 \%$ & $3.36 \%$ & $0.38 \%$ & $2.69 \%$ & $2.00 \%$ & $0.31 \%$ & $0.28 \%$ \\
\hline \multicolumn{7}{c}{ Proportions of nasal polyps in the subgroups with co-existing allergic and non-allergic conditions } \\
\hline $3.36 \%$ & $3.37 \%$ & $4.41 \%$ & $3.02 \%$ & $3.39 \%$ & $5.45 \%$ & $4 \%$ \\
$(n=21)$ & $(n=14)$ & $(n=3)$ & $(n=15)$ & $(n=10)$ & $(n=3)$ & $(n=2)$ \\
\hline
\end{tabular}

B. Odds ratio for risk factors for multimorbidity with nasal polyps

\begin{tabular}{lccccccc}
\hline Risk factors & NAR and $B A$ & NAR and AD & BA and AD & BA and AR & AD and AR & NAR and BA and AD & AR and BA and AD \\
\hline OR & 3.35 & 6.09 & 4.38 & 2.93 & 4.15 & 5.51 & 3.9 \\
\hline $95 \%$ Cl for OR & $2.12-5.31$ & $3.4-10.93$ & $1.36-14.05$ & $1.72-4.99$ & $2.15-7.99$ & $1.71-17.8$ & $0.94-16.16$ \\
\hline$P$-value & $<0.001$ & $<0.001$ & 0.035 & $<0.001$ & $<0.001$ & 0.02 & 0.1 \\
\hline
\end{tabular}

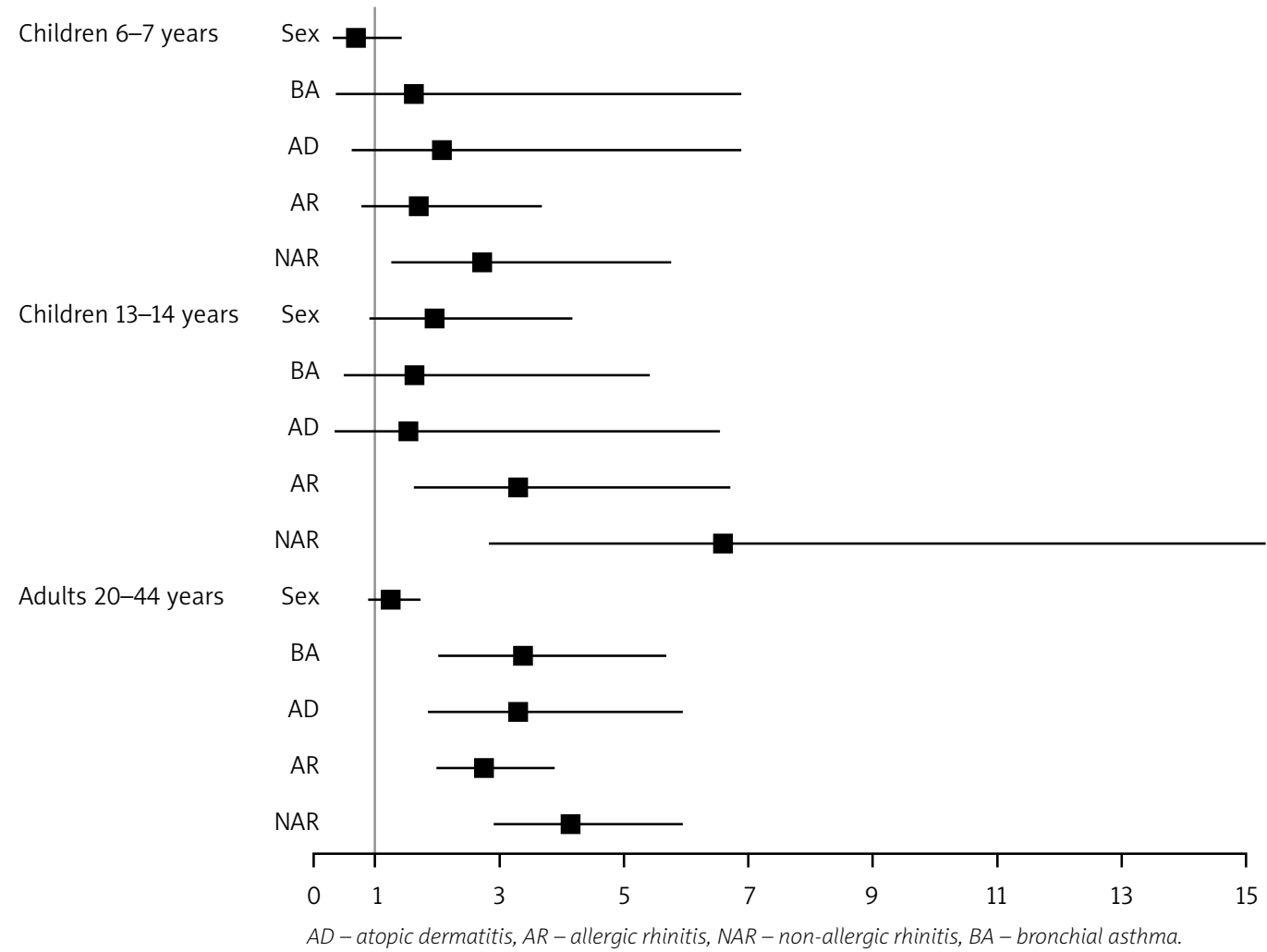

Figure 3. Odds ratio for the factors affecting the prevalence of NP, stratified by respondent characteristics

have NP?, while disregarding the symptomatology specific to CRSWNP, which can be indicative of its diagnosis. It is also prudent to note the questionnaire-based nature of this study as its limitation. Nonetheless, due to the fact that nasal polyps pose relatively few diagnostic difficulties, both to physicians and patients themselves, and considering the general familiarity with this term, for the purpose of our study we assumed that no respondents would submit a false positive response to the question Do you have NP? without a prior medical diagnosis. On the other hand, a separate and severe issue in epidemiological assessment is that of undiagnosed NP among individuals who did not seek medical assistance due to nasal symptoms. Workman et al. assessed the applicabili- 
ty of questionnaires in self-diagnosing CRS: the questionnaires, completed by the patients themselves, included information on self-reported CRS status, symptoms, and demographic data. The sensitivity of self-reported CRS diagnoses was $84 \%$, the specificity was $82 \%$, and the negative predictive value was $97-99 \%$. The authors concluded that such assessments may be a useful and relatively inexpensive screening method, and provide a reliable estimate of CRS prevalence, especially in large-cohort studies [7]. The main limitation of our study was the demography of respondents, restricted to three age ranges of 6-7, 13-14, and 20-44 years; although commonly employed in epidemiological research on allergic conditions, they are a subpar choice considering the nature of CRSWNP, the incidence of which displays a dramatic increase with age and peaks in patients aged 50 to 80 years old [2, 14-17].

Our study revealed a prevalence of nasal polyps of $1.1 \%$, derived from the evaluation of 18,458 respondents, 204 of whom reported NP. In light of the current literature, based on subjective questionnaire-based assessments and objective anterior rhinoscopy, the prevalence of NP is estimated at $1-4 \%$ of the general population [2]. Post-mortem endoscopic findings indicate a decisively more common (up to $32-42 \%$ ) incidence of polyp-like mucosal lesions in the nose and paranasal sinuses. Still, it is important to note that those predominantly small lesions rarely develop beyond a preclinical stage [18]. Based on a population-based study in the Netherlands, in which the mean age of participants was 47.9. Lange et al. reported a prevalence of chronic rhinosinusitis of $9 \%$, and a prevalence of polyps of $4 \%$ [15]. Portenko's questionnaire-based study of 6748 respondents, including children and adults, carried out in the Kalinin region in Russia, revealed a 1.3\% prevalence of NP [19]. A questionnaire-based study by Hedman et al. conducted among 4300 adult Finnish respondents (aged 18 to 65 years) showed a $4.3 \%$ prevalence of NP [5].

The typical age of onset of NP is 50-60 years, with the condition found more commonly in men $[1,2]$. According to our study, $1.5 \%$ of the evaluated 20-44-yearolds reported NP, and the prevalence displayed a clear increase with age. Taking into account the adult age group analysed in our study, it is essential to emphasise that they did not constitute a representative part of the population most typically afflicted with NP. Consistent with the findings of other researchers, our study showed NP to be very rare in children, affecting $0.6 \%$ of both $6-7$-yearolds and 13-14-year-olds. In comparison, Larsen and Tos evaluated 252 patients with NP and reported no cases of NP among children under the age of 10 years, with only 11 cases in 10-19-year-olds. Despite their rare incidence, nasal polyps remain the most common tumour of the sinonasal tract in this population, diagnosed in approximately $0.1-0.5 \%$ of minors. The discovery of NP in a child should always increase clinical wariness and lead to further diagnostic investigations directed at systemic con- ditions, predominantly cystic fibrosis, but also including primary ciliary dyskinesia, NSAID hypersensitivity, and primary immunodeficiencies [1, 2, 15, 20].

Our study revealed a higher prevalence of NP in females in the 6-7-year-olds group and in males in the 13-14-year-olds group, as well as in adult males. These differences, however, were not significant. With regard to various studies: Larsen and Tos reported a higher overall NP prevalence in men [18]; according to Stevens et al., males are affected by CRSWNP more frequently, while females display a more severe course of the condition [2]; the predominance of males in the patient population affected by NP is nullified when the condition co-occurs with BA, especially BA with NSAID hypersensitivity, thus rendering the prevalence of NP to be comparable in both sexes; considering the cases of the ASA triad, which consists of NP, BA, and aspirin intolerance, the prevalence of $N P$ is higher in females than in males $[1,2,15]$. Approximately $5 \%$ of patients are diagnosed with antrochoanal polyps, which correlates with a lower mean age in this group, as opposed to other patients with NP [21].

Nasal polyps rarely constitute an isolated pathology because there are multiple conditions known to frequently coexist with NP. Regarding the inflammatory nature of said conditions, it may be postulated that NP is associated with the phenomenon of multimorbidity through a common mechanism of pathogenesis [8, 9]. Taking into account both the relevant literature and our own research, there is a clear association between BA and NP [1]. Our study demonstrated higher incidence rates of NP in patients with BA, the occurrence of which increased the risk of NP by 3.5 fold. According to a variety of sources, it is estimated that $20 \%$ to $72 \%$ of patients with NP may have symptomatic BA, while symptomatic BA combined with NSAID hypersensitivity may occur in up to $25 \%$ of patients $[1,4,15,19,21]$. Stevens et al. draw attention to the fact that approximately $88 \%$ of patients with BA exhibit radiographic evidence of sinusitis [2]. NSAID hypersensitivity is a poor prognostic factor forecasting a high tendency of NP recurrence. On the other hand, effective treatment of CRSWNP may in turn contribute towards a better management of lower respiratory tract symptoms [1]. Other conditions commonly associated with NP include: cystic fibrosis, primary ciliary dyskinesia (Kartagener syndrome), Churg-Strauss syndrome, allergic fungal rhinosinusitis, and bronchiectasis. To date, no definitive consensus regarding the relationship between NP and allergic condition has been reached. In our study AR increased the risk of developing NP 2.5 fold ( $p<0.001$ ), while patients with NAR had a nearly 6-fold higher risk ( $p<0.001)$, compared to those without AR. Furthermore, the prevalence of NP in the evaluated population was higher among patients with concomitant AD. There is a lack of comparable data in contemporary literature that would assess the comorbidity of nasal polyps and atopic dermatitis. A possible explanation could stem from the superantigens theory and 
the role of Staphylococcus aureus. Bachert et al., Schubert, and Bernstein et al. were the first to propose a correlation between $S$. aureus infection and the development of nasal polyps, particularly in association with asthma. Researchers hypothesised that $S$. aureus exotoxins may act as superantigens, inducing local IgE production, which could be associated with the inflammatory changes of the sinonasal mucosa observed in CRSwNP [22-24]. The aforementioned hypothesis was recently discussed in a metaanalysis by Ou et al., as well as a paper by Vickery et al., who concluded that $S$. aureus superantigens may be a risk factor for both the persistence and the severity of chronic rhinosinusitis with nasal polyps, with the detection of S. aureus-superantigens related to poor outcomes and overall CRSWNP severity $[25,26]$.

The presence of $S$. aureus has also been examined in relation to atopic dermatitis. The pathogen was reported to selectively colonise the lesional skin of patients with $A D$, while being absent from the skin of the majority of healthy individuals. The increase in S. aureus colonisation correlates with severity and disease flares of AD due to S. aureus toxins acting as superantigens that promote inflammatory reactions in the skin. Nevertheless, the precise role of $S$. aureus in the pathogenesis of $A D$ remains unclear [27].

Pearlman et al. specifically accentuated the weakness of the AR and NP relationship, indicating that the rates of NP in AR patients (1.5-1.7\%) are comparable to those in the general population [21]. However, the role of atopy in the development of NP has been the subject of extensive and long-lasting discussion. Various study results seem to disprove the direct role of IgE-mediated allergy, a systemic condition with positive skin-prick tests and the presence of specific serum IgE, in the pathogenesis of NP, further postulating that such allergies are no more common in patients afflicted with NP than in the general population. Recent years have seen an interesting new motion emerge among scholars, discussing the role of local hypersensitivity and IgE production limited to the sinonasal mucosa, and their subsequent role in the pathogenesis of NP, including the production of specific IgE induced by exposition to S. aureus superantigens [28]. The co-occurrence of systemic allergic inflammation and nasal polyps certainly has an unfavourable effect on the course of the latter condition by aggravating the inflammatory process, impeding and complicating treatment, and increasing recurrence rates $[1,4,6,19,29,30]$.

Our study revealed NP to be significantly less common in rural areas, as opposed to urban ones, with $13(0.6 \%)$ of the 2034 rural respondents and 191 (1.1\%) of the 16,424 urban respondents reporting the condition ( $p=0.043)$. Portenko's results also confirm a higher prevalence of NP among metropolitan residents [18]. A possible explanation might stem from the impact of environmental and lifestyle factors on the development of NP. With regards to this subject, our study did not identify exhaust fumes exposure as a factor linked to NP prevalence; we did, however, observe higher prevalence of NP in respondents who smoked tobacco. These findings are consistent with the data presented by other researchers [1].

\section{Acknowledgments}

The ECAP study was part of research project No. 6 PO5 2005 C/06572 "Implementation of the system for the prevention and early detection of allergies in Poland", commissioned by the Polish Minister of Health.

\section{Conflict of interest}

The authors declare conflict of interest.

\section{References}

1. Fokkens WJ, Lund VJ, Mullol J, et al. EPOS 2012: European position paper on rhinosinusitis and nasal polyps 2012. Rhinology 2012; 23: 1-298.

2. Stevens WW, Schleimer RP, Kern RC. Chronic rhinosinusitis with nasal polyps. J Allergy Clin Immunol Pract 2016; 4: 565-72.

3. Settipane GA. Epidemiology. In: Nasal Polyps: Epidemiology, Pathogenesis and Treatment. Settipane GA, Lund VJ, Bernstein JM, Tos M (eds). OceanSide Publication Inc. Rhode Island, 1997; 17-24.

4. Klossek JM, Neukirch F, Pribil C, et al. Prevalence of nasal polyposis in France: a cross-sectional, case-control study. Allergy 2005; 60: 233-7.

5. Hedman J, Kaprio J, Poussa T, et al. Prevalence of asthma, aspirin intolerance, nasal polyposis and chronic obstructive pulmonary disease in a population-based study. Int J Epidemiol 1999; 28: 717-22.

6. Zhang Y, Gevaert E, Lou H, et al. Chronic rhinosinusitis in Asia. J Allergy Clin Immunol 2017; 140: 1230-9.

7. Workman AD, Parasher AK, Blasetti MT, et al. Accuracy of self-reported diagnosis of chronic rhinosinusitis. Otolaryngol Head Neck Surg 2019; 160: 556-8.

8. Tan BK, Chandra RK, Pollak J, et al. Incidence and associated premorbid diagnoses of patients with chronic rhinosinusitis. J Allergy Clin Immunol 2013; 131: 1350-60.

9. Bousquet J, Anto JM, Wickman M, et al. Are allergic multimorbidities and IgE polysensitization associated with the persistence or re-occurrence of foetal type 2 signalling? The MeDALL hypothesis. Allergy 2015; 70: 1062-7.

10. Raciborski F, Bousqet J, Namysłowski A, et al. Dissociating polysensitization and multimorbidity in children and adults from a Polish general population cohort. Clin Transl Allergy 2019; 9: 4.

11. Gelardi M, Iannuzzi L, Tafuri S, et al. Allergic and non-allergic rhinitis: relationship with nasal polyposis, asthma and family history. Acta Otorhinolaryngol Ital 2014; 34: 36-41.

12. European Community Respiratory Health Survey: Variations in the prevalence of respiratory symptoms, self-reported asthma, and use of asthma medication in the European Community Respiratory Health Survey. Eur Respir J 1996; 9 : 687-95.

13. The International Study of Asthma and Allergies in Childhood (ISAAC) Steering Committee: worldwide variation in 
prevalence of symptoms of asthma, allergic rhinoconjunctivitis, and atopic eczema. Lancet 1998; 351: 1225-32.

14. Meltzer EO, Hamilos DL, Hadley JA, et al. Rhinosinusitis: establishing definitions for clinical research and patients' care. J Allergy Clin Immunol 2004; 114: 155-212.

15. Lange B, Holst R, Thilsing T, et al. Quality of life and associated factors in persons with chronic rhinosinusitis in the general population: a prospective questionnaire and clinical cross-sectional study. Clin Otolaryngol 2013; 38: 474-80.

16. Larsen K, Tos M. The estimated incidence of symptomatic nasal polyps. Acta Otolaryngol 2002; 122: 179-82.

17. Johansson L, Akkerlund A, Holmberg K, et al. Prevalence of nasal polyps in adults: the Skövde population-based study. Ann Otol Rhinol Laryngol 2003; 112: 625-9.

18. Larsen PL, Tos M. Site of origin of nasal polyps. Transcranially removed naso-ethmoidal blocks as a screening method for nasal polyps in autopsy material. Rhinology 1995; 33: 185-8.

19. Portenko GM. Prevalence of nasal polypous rhinosinusitis among the population. Vestn Otolaringol 1989; 1: 52-4.

20. Workman AD, Parasher AK, Blasetti MT, et al. Accuracy of self-reported diagnosis of chronic rhinosinusitis. Otolaryngol Head Neck Surg 2019; 160: 556-8.

21. Pearlman AN, Chandra R, Conley DB, Kern RC. Epidemiology of nasal polyps. In: Nasal Polyposis: Pathogenesis, Medical and Surgical Treatment. Onerci TM, Ferguson BJ (eds.). Springer, Berlin Heidelberg 2010; 11: 9-15.

22. Bachert C, Gevaert P, Holtappels G, et al. Total and specific IgE in nasal polyps is related to local eosinophilic inflammation. J Allergy Clin Immunol 2001; 107: 607-14.

23. Schubert MS. A superantigen hypothesis for the pathogenesis of chronic hypertrophic rhinosinusitis, allergic fungal sinusitis, and related disorders. Ann Allergy Asthma Immunol 2001; 87: 181-8.

24. Bernstein JM, Ballow M, Schlievert PM, et al. A superantigen hypothesis for the pathogenesis of chronic hyperplastic sinusitis with massive nasal polyposis. Am J Rhinol 2003; 17: 321-6.

25. Ou J, Wang J, Xu Y, et al. Staphylococcus aureus superantigens are associated with chronic rhinosinusitis with nasal polyps: a meta-analysis. Eur Arch Otorhinolaryngol 2014; 271: 2729-36.

26. Vickery TW, Ramakrishnan VR, Suh JD. The role of Staphylococcus aureus in patients with chronic sinusitis and nasal polyposis. Curr Allergy Asthma Rep 2019; 19: 21.

27. Yamazaki Y, Nakamura Y, Núńez G. Role of the microbiota in skin immunity and atopic dermatitis. Allergol Int 2017; 66: 539-44.

28. Bachert C, Gevaert E. Advances in rhinitis and rhinosinusitis in 2015. J Allergy Clin Immunol 2016; 138: 1277-83.

29. Huvenne W, van Bruaene N, Zhang N, et al. Chronic rhinosinusitis with and without nasal polyps: what is the difference? Curr Allergy Asthma Rep 2009; 9: 213-20.

30. Guilemany JM, Angrill J, Alobid I, et al. United airways again: high prevalence of rhinosinusitis and nasal polyps in bronchiectasis. Allergy 2009; 64: 790-7. 